\title{
What about STEMI in COVID-19 Women?
}

\author{
Patrizia Presbitero $^{1}$, Carla Lucarelli $^{1}$, Marco Mennuni $^{2}$, and Emanuela Piccaluga ${ }^{3}$ \\ ${ }^{1}$ IRCCS Humanitas Clinical and Research Center, Clinical and Interventional Cardiology Department, Rozzano, Milan, Italy \\ ${ }^{2}$ Novara Hospital, Clinical and Interventional Cardiology Department, Milan, Italy \\ ${ }^{3}$ Niguarda Ca 'Granda Hospital, Clinical and Interventional Cardiology Department, Milan, Italy
}

\begin{abstract}
There is no doubt that in the last 2 months during COVID-19 pandemic a smaller number of ST-elevation Myocardial Infarction (STEMI) and Stroke arrived to cardiological and neurological departments. The pathophysiology of STEMI in COVID-19 women is not fully understood; it could be thrombus recanalization, catecholamine storm or Type 2 Myocardial infarction in case of severe respiratory distress or direct myocardial damage (viral myocarditis). Because most of them have normal coronary arteries an invasive strategy with coronary angiography is important to rule out atherosclerotic severe coronary disease.
\end{abstract}

Keywords: STEMl; Left ventricle; Anxiety; Papillary muscle rupture; Intraventricular thrombus

\section{Introduction}

The data on this, at least for STEMI are confirmed in most of the countries: the reduction varies from 15 up to $50 \%[1,2]$. Most Cardiology Societies and Interventional Cardiologists are ascribing this phenomenon to the fear of patients to be infected when coming to casualty departments. This is corroborated also by data reporting an increase in late complications of STEMI that we were not used to see anymore: thick intracoronary thrombi (high thrombotic burden), left ventricle or papillary muscle rupture, intraventricular thrombus. However, fear of infection cannot be the only explanation because this reduction occurs also in country where lock down and the diffuse anxiety toward the infection were not applied, like Sweden.

\section{Literature Review}

On the contrary, out of hospital cardiac arrests increased by $58 \%$ in Lombardia in comparison with the previous year during the same period [3]. The cumulative incidence was strongly associated with the cumulative incidence of COVID-19: $77 \%$ of the increase in cases had diagnosis of COVID-19. Therefore, it is unknown whether an arrhythmic event, a sudden occlusion of a coronary artery, catecholamine storm, a stroke or pulmonary embolism due to high thrombogenicity were responsible for these deaths. The reduction in STEMI could be explained by the increase in COVID-19 related deaths: the two diseases are competing for the same age and comorbidity people. A further explanation could also be the reduction in the trigger for ischemic events: less travels, less work stress, lower pollution difficult to quantify.

How these considerations apply to women and STEMI during COVID-19 pandemic? We know now that the number of affected cases is the same in males and females (Global Health 5050) with some variations between countries. However, in almost all countries, data show that, once infected with COVID-19, the mortality rate is higher for men compared to women: in
Italy $63 \%$ versus $37 \%$, in Spain $58 \%$ versus $42 \%$, in Germany $57 \%$ versus $43 \%$. A Chinese study including 1056 patients reported that mortality in men is 2,5 times higher than in women. A similar tendency with mortality significantly higher in men than in women was reported for SARS in 2003 [4].

In summary, in most countries available data indicates that men have being upwards of $50 \%$ more likely to die following diagnosis than women. It is well known that among mammals, sex related differences exist in the innate and adaptative immune response, determining different susceptibility to infectious disease, with a clear bias towards the male gender proven in some viral infections such as MERS-CoV (Middle East Respiratory Syndrome Corona Virus) [5]. Prof. Philip Goulder (Immunologist, Oxford University) said that women's immune response to the virus is stronger, "The protein by which viruses like coronavirus are sensed is encoded on the $X$ chromosome, and as a result this protein is expressed at twice the dose in many immune cells in females compared to males". Probably, there are also gender lifestyle differences: for example, in China $50 \%$ of males are smoking against $5 \%$ of women. In Italy younger women (20-49 years) are affected as men and this is the range in which female employment rate is closer to the male rate, mainly in health and education.

Despite quarantine, with the psychological stress related to lock down and the proved increase in domestic violence $(+30 \%$ in France, $+75 \%$ in Australia and $+50 \%$ in US), the incidence rate of STEMI in women did not increase compared with the previous years, as reported in recent series [6].

Stefanini et al. [7] recently reported a series of 28 STEMI in COVID-19 patients, 8 of which were women: 5 of them $(62 \%)$ did not have coronary artery diseases which is much more that what we see in STEMI female patients (3\%) [8]. Another report showed (AS Petronio, case report submitted) coronary extensive thrombosis with otherwise normal coronary arteries.

We reviewed data of STEMI during COVID-19 pandemic in March-April 2020 compared with the same period of 2019 from 3 hospitals in Piemonte Lombardia (Figure 1), as in most hospitals in Countries with high incidence of

*Address for Correspondence: Patrizia Presbitero, IRCCS Humanitas Clinical and Research Center, Clinical and Interventional Cardiology Department, Rozzano, Milan, Italy, Tel: +393355361638; E-mail: patrizia.presbitero@humanitas.it 
the disease a $30 \%$ overall reduction in STEMI was noticed. Reduction was more marked in women ( $59 \%$ reduction) compared with men (12\% reduction) $(p=0.04$, Fisher Exact). These data confirm the well-known gender gap in acute coronary syndrome, according which women tend to delay in seeking medical care [9].

A small minority of patients had COVID-19. Only two female patients were found to have no coronary artery disease. Both were admitted to hospital with a severe form of the disease requiring orotracheal intubation, none of them had STEMI related symptoms.

As in Stefanini series the S-T elevation, with high troponin levels and regional wall motion abnormalities in female STEMI with COVID-19 was found during the hospitalization for severe respiratory failure. Despite the severe form of the disease and the myocardial damage, the survival was good. The two COVID-19 positive men were admitted for chest pain had coronary artery disease. One of them had a complicated clinical course; the other was discharged at home three days after the procedure

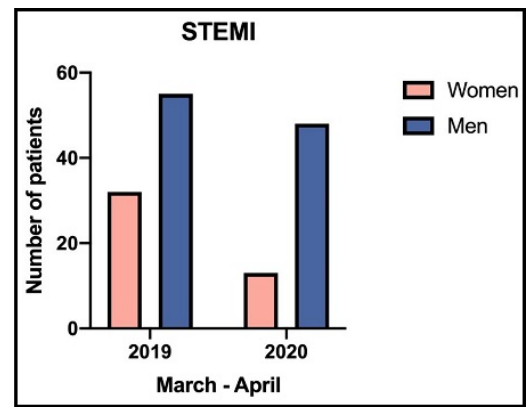

Figure 1. Number of admissions for STEMI during COVID-19 for women and men during March-April 2019 and 2020.

\section{Discussion and Conclusion}

Finally, less women than men suffer severe COVID-19 inflammatory reactions. The less severe clinical presentation, with lower incidence of venous and arterial thrombosis, could possibly explain the lower number of STEMI in COVID-19 women. Fewer myocardial infarctions in women than in men during COVID-19 pandemic could be not only explained by the fear of being infected in busy casualty departments, but also to the more important role in families during quarantine, making difficult to leave home.

The pathophysiology of STEMI in COVID-19 women is not fully understood; it could be thrombus recanalization, catecholamine storm or Type 2 Myocardial infarction in case of severe respiratory distress or direct myocardial damage (viral myocarditis). Because most of them have normal coronary arteries an invasive strategy with coronary angiography is important to rule out atherosclerotic severe coronary disease.

\section{Conflicts of Interest}

Authors have no conflict of interest to declare.

\section{References}

1. Solomon, Matthew D., Edward J. McNulty, Jamal S. Rana, and Thomas K. Leong, et al. "The Covid 19 Pandemic and the Incidence of Acute Myocardial Infarction." N Engl J Med (2020).

2. Rodríguez-Leor, Oriol, Belén Cid-Álvarez, Soledad Ojeda, and Javier MartínMoreiras, et al. " Impact of the COVID-19 pandemic on care activity in interventional cardiology in Spain." REC Interv Cardio/2 (2020): 82-89.

3. Baldi, Enrico, Giuseppe M. Sechi, Claudio Mare, and Fabrizio Canevari, et al. "Out-Of-Hospital cardiac arrest during the Covid-19 outbreak in Italy." N Eng/ $J \operatorname{Med}(2020)$

4. Jin, Jian-Min, Peng Bai, Wei He, and Fei Wu, et al. "Gender differences in patients with COVID-19: Focus on severity and mortality." Front Public Health 8 (2020): 152.

5. Klein, Sabra L., and Katie L. Flanagan. "Sex differences in immune responses." Nat Rev Immuno/16 (2016): 626-638.

6. De Rosa, Salvatore, Carmen Spaccarotella, Cristina Basso, and Maria Pia Calabrò, et al. "Reduction of hospitalizations for myocardial infarction in Italy in the COVID-19 era." Eur Heart J(2020).

7. Stefanini, Giulio G., Matteo Montorfano, Daniela Trabattoni, and Daniele Andreini, et al. "ST-elevation myocardial infarction in patients with COVID-19: Clinical and angiographic outcomes." Circulation (2020).

8. Da Costa, A., K. Isaaz, E. Faure, and S. Mourot, et al. "Clinical characteristics, aetiological factors and long-term prognosis of myocardial infarction with an absolutely normal coronary angiogram; a 3-year follow-up study of 91 patients." Eur Heart J22 (2001): 1459-1465.

9. Haider, Ahmed, Susan Bengs, Judy Luu, and Elena Osto, et al. "Sex and gender in cardiovascular medicine: presentation and outcomes of acute coronary syndrome." Eur Heart J41 (2020): 1328-1336.

How to cite this article: Patrizia Presbitero, Carla Lucarelli, Marco Mennuni, and Emanuela Piccaluga. "What about STEMI in COVID-19 Women?". J Cardiovasc Dis Diagn 8 (2020) doi: 10.37421/jcdd.2020.8.407 